\title{
État des connaissances sur les échanges entre l'eau, les matières en suspension et les sédiments des principaux radionucléides rejetés en eau douce par les centrales nucléaires
}

\author{
J. GARNIER-LAPLACE*, V. FOURNIER-BIDOZ*, J.P. BAUDIN**
}

(Manuscrit reçu le 22 novembre 1995, révisé le 10 octobre 1996, accepté le 22 octobre 1996)

RÉSUMÉ Dans le domaine des eaux douces, un très grand nombre de travaux ont été réalisés pour caractériser les échanges des radionucléides entre phases solide et liquide constituant les compartiments physiques des écosystèmes naturels. Ces échanges dépendent des conditions physiques, chimiques et biologiques régnant dans le milieu. Généralement, le concept de Kd ou coefficient de distribution, est utilisé pour quantifier la répartition du radionucléide entre phases dissoute et particulaire. Les principaux paramètres environnementaux influençant la valeur du Kd sont les suivants : temps de contact, concentration en matières en suspension, nature et granulométrie de la phase solide (plus particulièrement pour le césium, le cobalt, le manganèse, l'argent, l'iode), concentration en isotopes stables des phases solide et liquide (césium, cobalt, manganèse, argent), conditions d'oxydoréduction des deux phases (césium, manganèse), composition minérale de la phase liquide (césium), pH de l'eau (cobalt, manganèse, argent), composés organiques dissous (cobalt, antimoine, iode). Ainsi, en liaison avec l'influence de ces divers paramètres, la gamme de variation du Kd est très vaste pour chaque radionucléide. Cette gamme peut être réduite, pour un écosystème à un moment donné de son cycle hydrologique, si les valeurs in situ des paramètres majeurs influençant les échanges entre phases sont connus. La réalisation d'expériences en laboratoire et/ou de terrain permet de déterminer la valeur de Kd adaptée à la situation étudiée.

ABSTRACT In the field of freshwater environment, many studies have been carried out to characterize the interaction of radionuclides with the solid and liquid phases that constitute the physical components of natural ecosystems. These exchanges depend upon physical, chemical and biological conditions characterizing the system. To quantify the distribution of the radionuclide between dissolved and particulate forms, a ratio expressed as Kd or distribution coefficient, is generally used. The major environmental parameters that influence the Kd values are : contact time and suspended matter concentrations, quality and granulometry of the solid phase (especially for cesium, cobalt, manganese, silver, iodine), stable

\footnotetext{
* Institut de protection et de sureté nucléaire, Département de protection de l'environnement et des installations, Laboratoire de radioécologie continentale, IPSN, 13108 St-Paul-lezDurance, Cedex, France.
}

** Centre national de la recherche scientifique, même adresse. 
isotope concentrations in the liquid and solid phases (cesium, cobalt, manganese, silver), oxydoreduction conditions in the two phases (cesium, manganese), mineral composition of the liquid phase (cesium), pH of water (cobalt, manganese, silver), presence of dissolved organic ligands (cobalt, antimony, iode). As a result, for each radionuclide, the range of the distribution coefficient is considerable. However, for a given ecosystem at a given period of its hydrological cycle, this range can be reduced if the values of the main environmental parameters that influence the Kd values are known. The site-specific values of $\mathrm{Kd}$ can also be determined by experiments carried out under appropriate conditions in laboratory or in situ.

\section{Introduction}

Ces dernières années, les recherches en radioécologie ont essentiellement concerné les milieux estuariens et marins considérés comme les exutoires des cours d'eau où s'effectuent la majorité des rejets d'effluents liquides d'installations nucléaires. En France, l'implantation le long des fleuves et rivières, de quinze sites électronucléaires sur dix neuf en fonctionnement, impose de mettre l'accent sur les eaux douces continentales. D'une manière générale, celles-ci présentent un grand intérêt, non seulement en raison de leur utilisation par l'homme, mais aussi parce qu'elles peuvent constituer autant d'écosystèmes soumis aux risques d'accidents nucléaires comme en témoignent les réservoirs en cascade du Niepre qui posent encore actuellement des problèmes de contamination consécutivement à l'accident de Tchernobyl. De la même façon que pour d'autres polluants, la concentration en radionucléides dans les eaux de surface est contrôlée par un ensemble de mécanismes physiques, chimiques et biologiques incluant l'aspect hydraulique (advection, dispersion), la décroissance radioactive, les interactions diverses avec les particules physiques (matières en suspension et sédiments), elles-mêmes soumises aux lois du transport solide (remise en suspension, dépôts, charriage...). Le comportement d'un radionucléide introduit dans un écosystème et sa spéciation à un instant donné vont dépendre d'un ensemble d'interactions entre les différents compartiments en présence : colonne d'eau, matières en suspension, sédiments de surface, sédiments profonds, eau interstitielle des sédiments, compartiments biotiques. Ces interactions sont régies par les conditions physiques et chimiques du milieu récepteur, entre les formes initialement rejetées et les composants présents dans le milieu. La figure 1 représente les différentes formes d'existence d'un élément chimique (isotopes stables et radioactifs de même numéro atomique) dans un système naturel d'eau douce, avec leurs évolutions possibles.

Dans cet article, un état des connaissances actuelles est dressé sur les échanges en eau douce, entre phases liquide et particulaire, des huit radionucléides entrant dans la composition hors ${ }^{3} \mathrm{H}$ des effluents liquides de centrales électronucléaires françaises en fonctionnement normal ${ }^{134} \mathrm{Cs},{ }^{137} \mathrm{Cs},{ }^{58} \mathrm{Co}$, $\left.{ }^{60} \mathrm{Co},{ }^{110 \mathrm{~m}} \mathrm{Ag},{ }^{125} \mathrm{Sb},{ }^{54} \mathrm{Mn},{ }^{131} \mathrm{I}\right)$. Cet article constitue un recueil des connais- 


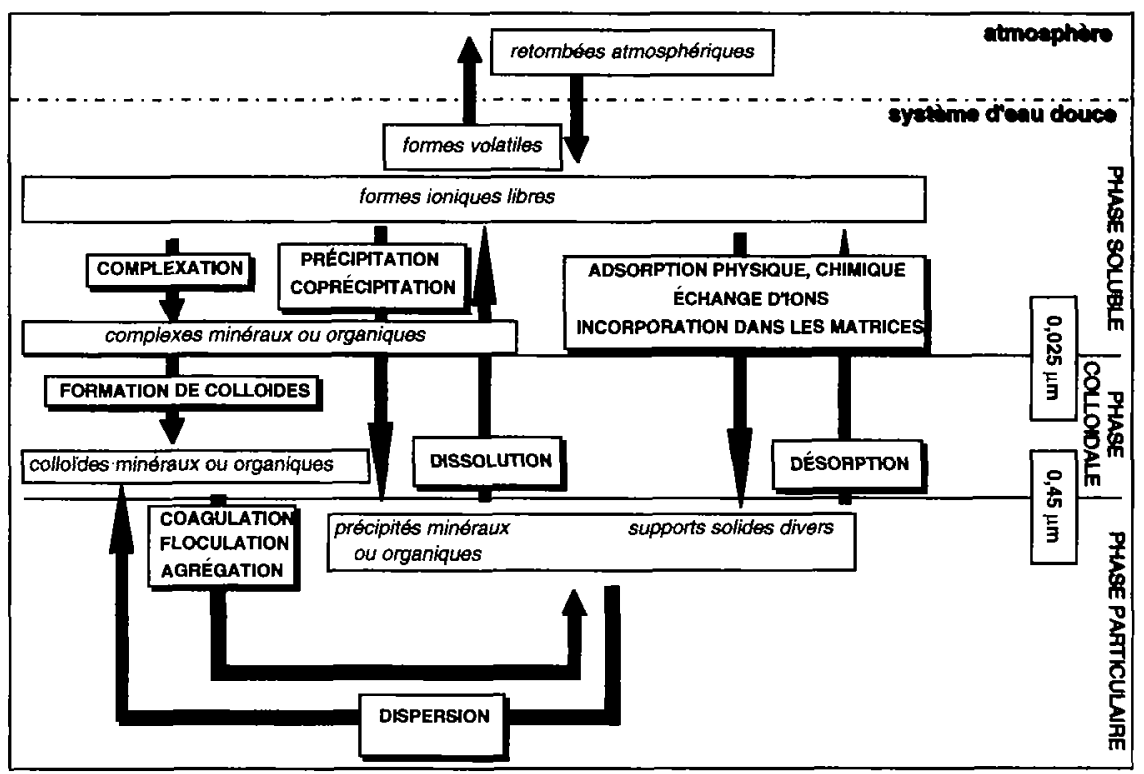

Fig. 1. - Phases et processus déterminant le comportement d'un élément chimique dans un système d'eau douce naturel.

Phases and mechanisms characterizing the radionuclide behaviour in a natural freshwater ecosytem.

sances préliminaires nécessaires à l'engagement de toute étude d'échanges de radionucléides entre phases dissoute et particulaire, constitué sur la base de la compilation des principales publications existant en eau douce et dominé par l'idée générale que le Kd n'est pas une constante pour un radionucléide donné, mais est spécifique d'un site à un instant donné de son cycle hydrologique. L'exposé des données et conclusions est fondé sur l'analyse d'environ 140 articles ou ouvrages dont la citation n'a pas été reprise ici pour des raisons de longueur. L'ensemble des informations figure au sein d'une étude bibliographique réalisée par Founier-Bidoz et Garnier-Laplace (1994). Après avoir énoncé les définitions relatives au concept du Kd ou coefficient de distribution, et les mises en garde quant aux limites d'emploi de ce paramètre, ce bilan des connaissances sert de. base à la proposition d'une méthode d'étude dont l'objectif est de répondre à la question : quelle(s) valeur(s) de Kd utiliser pour quantifier les échanges des radionucléides entre phases liquide et particulaire au sein d'un écosystème donné ? 


\section{Concept du coefficient de distribution (Kd)}

La complexité des mécanismes d'échange entre les phases solide et liquide, au sein d'un écosystème donné, a conduit les scientifiques à adopter des modèles généraux pour décrire globalement les effets combinés se produisant dans le système étudié. Ainsi, les modèles empiriques décrivant l'état d'équilibre de la distribution d'un élément dans un système à plusieurs composants (modèles de type Freundlich, Langmuir...) sont communément utilisés même si leur emploi est souvent abusif et controversé. Il existe également des modèles décrivant les cinétiques de réaction, généralement construit sur des lois du premier ordre et des modèles chimiques bâtis sur les lois de la thermodynamique et sur la loi d'action de masse. Une revue des différents modèles existants a été réalisée par plusieurs auteurs (AIEA, 1985 ; Dzombak et Ali, 1993 ; Onishi et al., 1981).

\subsection{Définitions et limites d'utilisation}

En radioécologie, la démarche la plus fréquente consiste à utiliser des modèles plus ou moins complexes décrivant les principaux processus de sorption physique, chimique ou biologique, en faisant intervenir la notion de coefficient de distribution ou $\mathrm{Kd}$. Le terme de sorption est classiquement utilisé chaque fois que des processus d'adsorption (phénomène de surface) et d'absorption (phénomène de volume) sont impliqués. Le paramètre $\mathrm{Kd}$ créé pour quantifier la distribution d'un élément trace entre phases particulaire et dissoute, est le facteur de proportionnalité entre la concentration de l'espèce étudiée dans la phase solide $C_{\mathrm{s}}$ et sa concentration dans la phase liquide $C_{\mathrm{l}}$ :

$$
\mathrm{Kd}=\frac{C_{\mathrm{s}}}{C_{1}}\left(\text { en m }^{3} \mathrm{~kg} \mathrm{sec}^{-1}\right)
$$

À l'origine, ce concept a été développé à partir de la théorie de l'échange d'ions, imposant que les hypothèses relatives à son application soient vérifiées. En particulier, l'élément est présent à l'état de trace, l'équilibre entre les phases est établi et le phénomène est totalement réversible, décrit par un isotherme d'adsorption linéaire, ce qui n'est pas toujours le cas. Les conditions de non application de l'équilibre sont diverses dans les écosystèmes naturels : addition par l'homme de polluants dans le milieu, variations saisonnières des conditions physico-chimiques, modifications des temps de résidence des particules de diverses natures... L'utilisation du $\mathrm{Kd}$, s'il est défini comme une grandeur décrivant un seul état qui est l'équilibre, est donc limitante. La nécessité d'une approche cinétique est indiscutable et a été abordée par différents auteurs. Dans cet article, la terminologie de $\mathrm{Kd}$ ou coefficient de distribution sera employée comme mode d'expression du rapport de concentration entre phases liquide et solide à un instant donné, sans référence particulière à l'état d'équilibre sauf précision ( $\left.\mathrm{Kd}_{\text {équilibre }}\right)$ et sans donner d'informations sur les mécanismes ou les cinétiques d'échange mis en jeu. 
Le concept de $\mathrm{Kd}$ est utilisé par les radioécologistes géochimistes pour quantifier à la fois les phénomènes d'adsorption et de désorption. Ce dernier met en jeu deux mécanismes de base : le déplacement de l'élément chimique à partir du solide (rupture de liaison, changement d'hydratation de l'espèce liée) et le changement de formes physico-chimiques de l'espèce fixée (" dissolution »). Plusieurs phénomènes sont à l'origine de la désorption tels le changement de la concentration ionique de la phase liquide ou la modification de la température, du $\mathrm{pH}$ ou encore du potentiel d'oxydo-réduction. Les auteurs ont en général étudié les processus de désorption lors du passage de la phase solide en eau de mer et leurs principales conclusions sont énoncées, pour chaque radionucléide, dans le paragraphe traitant de l'influence de la salinité.

\subsection{Influence des paramètres environnementaux. Répercussion sur l'utilisation du Kd dans les modèles globaux de calcul de doses à l'homme}

$\mathrm{Vu}$ la complexité des phénomènes, la valeur du Kd pour un radionucléide est fonction d'un nombre important de paramètres définissant les conditions physiques, chimiques et biologiques régnant dans l'écosystème. Les paramètres environnementaux influençant la valeur du Kd sont répertoriés de manière non exhaustive dans le tableau I. Ainsi, même si la détermination du $\mathrm{Kd}$ par diverses techniques (mesures de terrain, techniques de laboratoire en milieu fermé (batch) agité ou statique, technique en couche mince, technique par dialyse, technique de sédimentation) est simple à appliquer, les valeurs obtenues pour un même élément s'inscrivent dans une gamme de variation étendue sur plusieurs ordres de grandeurs. L'utilisation d'une valeur donnée de $\mathrm{Kd}$ inadéquate par rapport au site étudié, dans un modèle global, qui va du scénario de rejet jusqu'au calcul sanitaire, pose alors la question du réalisme des valeurs estimées de la dose à l'homme. À titre d'illustration, Håkanson et Peters (1995) montrent dans le cas d'un modèle de simulation de la concentration en ${ }^{137} \mathrm{Cs}$ dans un poisson prédateur au sein d'un écosystème limnique (modèle VAMP) qu'une augmentation d'un facteur 10 de la valeur de $\mathrm{Kd}$ choisie entraîne une augmentation d'un facteur 8 de la concentration en ${ }^{137} \mathrm{Cs}$ estimée dans le poisson. La connaissance des paramètres présentés dans le tableau I permet de définir sous quelles conditions relatives aux phases liquide et solide, le Kd est obtenu et peut être utilisé. Lors d'une synthèse bibliographique, il est délicat d'analyser les valeurs de Kd rencontrées pour un même radionucléide sans connaître parfaitement les conditions, bien souvent omises par l'auteur, dans lesquelles ces valeurs ont été acquises. En plus des paramètres énoncés, il est, en tout premier lieu, indispensable de savoir si la valeur du Kd a été déterminée sur le terrain ou en laboratoire et si elle est relative au compartiment matières en suspension ou sédiments. En ce qui concerne ce dernier compartiment, l'approche de type Kd est largement discutable en raison de l'absence d'identification des phénomènes de type diffusion, migration en fonction de la profondeur, bioturbation, consolidation, sédimentation... 


\section{TABLEAU I}

Influence des paramètres environnementaux sur le coefficient de distribution des radionucléides. Répercussion(s) la(es) plus fréquente(s) d'une augmentation du paramètre cité sur la valeur du Kd : (+) augmentation du Kd ;

(-) diminution ; (=0) sans effet ; (?) non étudié ;

[ ]: autre(s) influence(s) parfois observée(s).

Influence of environmental parameters on the distribution coefficient value for radionuclides. Response(s) most frequently published to a rise of the given parameter: (+) increase of the Kd value ; $(-)$ decrease ; $(=0)$ without any effect ; [ ] other effect(s) sometimes observed.

\section{Paramétres eavironneneatau}

Paramètres hydrologiques :

Concentration en matières en suspension

Temps de contact

Paramètres relatifs à la phase solide:

Concentration en argiles

Concentration en matières organiques

Concentration en oxydes de fer

Concentration en oxydes de manganèse

Taille des particules

Paramètres relatifs à la phase liquide:

Concentrations en cations majeurs

Concentration en ligands minéraux

Concentration en matières organiques $\mathrm{pH}^{(2)}$

Potentiel redox

Température

Salinité

Concentration en élément chimique (radioactifs et stables)

Paramètres biologiques :

Présence et activité de microorganismes

$$
\begin{array}{cccccc}
+ & =0[+] & ? & + & ? & =0 \\
=0 & =0[+] & =0 & + & ? & =0[+] \\
=0 & =0[+] & ? & + & ? & + \\
=0 & =0[+] & ? & + & ? & + \\
- & - & - & ? & ? & +[-]
\end{array}
$$

$$
\begin{aligned}
& \begin{array}{llllll}
- & =0 & \quad & =0 & - & =0
\end{array} \\
& =0 \quad=0 \quad=0 \quad=0 \quad-\quad=0 \\
& =0 \quad+;-;=0 \quad=0 \quad=0 \quad-\quad- \\
& =0 \quad+;-\quad+\quad+;-;=0 \quad-\quad+[=0] \\
& =0+[-;=0]+\text { ? }+ \\
& =0[+] \quad ? \quad \text { ? } ? \text { ? }
\end{aligned}
$$

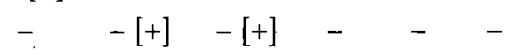

$$
\begin{aligned}
& -;=0 \quad-;=0 \quad-;=0 \quad-;=0 \quad \text { ? } \quad \text { ? }
\end{aligned}
$$

(1) L'influence des paramètres relatifs à la phase solide a été étudiée sur des sols de $\mathrm{pH}=4$ à 6 . Aucune publication n'existe sur le sujet en eau douce.

(2) L'influence du pH est testée dans la gamme de variation de ce paramètre dans les eaux naturelles, soit environ 4 à 8 . 


\section{Bilan des connaissances}

Les principales connaissances actuelles sont présentées de manière synthétique pour chaque radionucléide en soulignant les mécanismes et cinétiques mis en évidence ainsi que l'influence des principaux paramètres environnementaux. Le tableau I donne une vue globale de l'influence de ces paramètres sur l'évolution du $\mathrm{Kd}$. Dans la mesure du possible, cette influence est quantifiée par quelques exemples sélectionnés dans la littérature. Il est important de souligner que ces exemples ne peuvent constituer que des tendances à ne pas déconnecter du contexte spécifique de l'étude par laquelle elles ont été mises en évidence. Le tableau II donne les états de valence possibles dans les conditions régnant dans les écosystèmes d'eau douce et les informations disponibles sur la spéciation qui en découle. Afin de donner des ordres de grandeur des valeurs de $\mathrm{Kd}$, quelques exemples sont choisis parmi les études les plus intéressantes et les mieux renseignées sur le plan des protocoles expérimentaux (Tab. III). Une classification a été effectuée en trois catégories : valeurs issues d'études in situ, valeurs issues d'études en laboratoire et valeurs conseillées dans les modèles globaux de calcul de dose à l'homme. Un inventaire plus complet a été constitué (Fournier-Bidoz et Garnier-Laplace, 1994) et est repris dans le tableau III pour chacune des trois catégories précitées, soulignant la forte variabilité des Kd pour un radionucléide donné et le caractère de spécificité de la valeur de cette constante en fonction de la technique d'évaluation et de l'écosystème pour lequel elle a été estimée (constante dite "site-specific »).

\subsection{Césium}

\subsubsection{Mécanismes et cinétiques de sorption}

Lié à sa forme dissoute $\mathrm{Cs}^{+}$, dominante en eau douce, le mécanisme essentiel de sorption du césium est l'échange d'ions et concerne principalement les minéraux. Trois types de sites de fixation de sélectivité et réversibilité différentes, peuvent être distingués :

- les sites situés sur les surfaces des particules, non sélectifs, pour lesquels le césium fixé est facilement échangeable avec la plupart des cations ;

- les sites interfoliaires proches des bordures des particules pour lesquels le césium fixé peut être échangé seulement par des cations de taille et de charge voisine tels $\mathrm{K}^{+}$et $\mathrm{NH}_{4}{ }^{+}$. Ces sites offrent une sélectivité élevée pour la fixation des cations de faible énergie d'hydratation tels $\mathrm{Cs}^{+}, \mathrm{Rb}^{+}, \mathrm{NH}_{4}{ }^{+}$et $\mathrm{K}^{+}$;

- les sites interfoliaires à l'intérieur des particules, pour lesquels le césium est difficilement échangeable.

Deux cinétiques de sorption du césium sont mises en évidence lors d'expériences en laboratoire. L'essentiel de la sorption qui a lieu les premières heures ou premiers jours correspond aux sites de sorption de surface facilement accessibles. Les processus d'échange lents qui peuvent se poursuivre jusqu'à un mois, correspondent aux sites les moins accessibles situés à l'intérieur des minéraux argileux. 


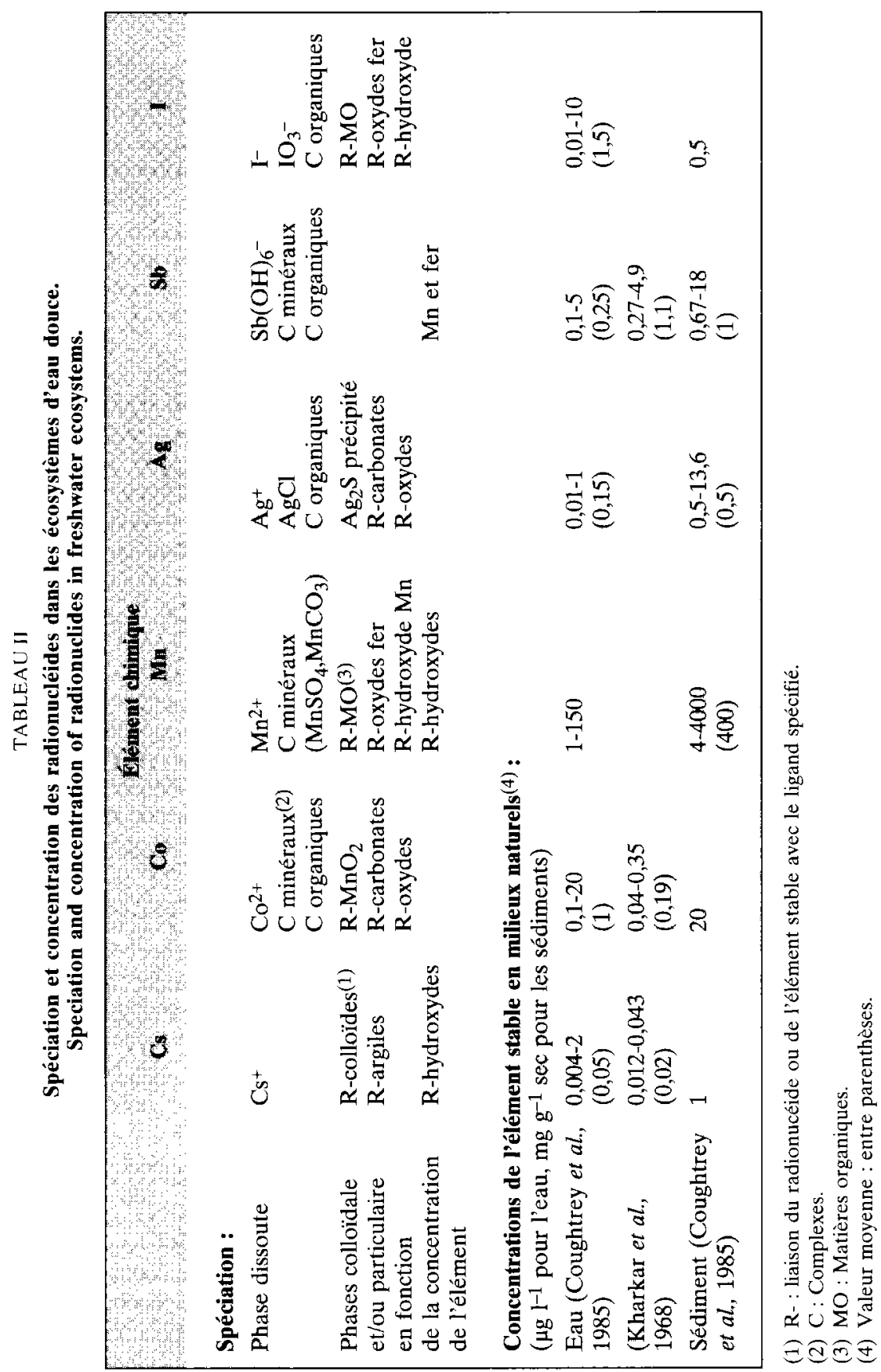




\subsubsection{Influence des paramètres environnementaux}

L'augmentation de la concentration des matières en suspension est généralement à l'origine d'une diminution des valeurs du $\mathrm{Kd}$. Une évolution inverse est parfois observée. À titre d'illustration, une étude in situ sur les matières en suspension du Pô (Italie) a montré qu'une augmentation de 10 à $100 \mathrm{mg} \mathrm{l}^{-1}$ de ce paramètre entraînait une diminution de la valeur du $\mathrm{Kd}$ de 30 à $5 \mathrm{~m}^{3} \mathrm{~kg} \mathrm{sec}^{-1}$ (Artioli et al., 1985). Les principaux composés solides responsables de la liaison du césium avec les sédiments ou les matières en suspension, sont les argiles, en particulier l'illite, c'est à dire des minéraux de fortes capacités d'échange cationique. Parmi les minéraux argileux, l'affinité du césium est par ordre décroissant : illite, biotite, hydrobiotite, montmorillonite, kaolinite avec une valeur du Kd divisée d'un facteur 100 environ depuis le premier matériau cité jusqu'au dernier (Onishi et al., 1981). A la suite d'expériences de contamination de sédiments en laboratoire, les résultats d'analyse élémentaire des fractions des échantillons étudiés obtenues par extraction sélective séquentielle ont permis de conclure que les enduits minéraux tels carbonates et oxydes ne contribuent pas significativement à la sorption du césium qui est gouvernée par l'affinité de l'élément pour les argiles. Généralement, la sorption du césium est plus importante pour les particules de petite taille en liaison avec la surface développée plus grande et un contenu plus élevé en minéraux de forte capacité de sorption tels les aluminosilicates. Par exemple, une augmentation d'un facteur 6 de la concentration en ${ }^{137} \mathrm{Cs}$ dans les sédiments du bas-Rhône a été observée en réponse à une augmentation de 5 à $90 \%$ des éléments fins (diamètre inférieur à $50 \mu \mathrm{m}$ ) dans la composition granulométrique des échantillons (Picat et al., 1979). Les cations monovalents $\left(\mathrm{K}^{+}, \mathrm{Na}^{+}\right.$et $\left.\mathrm{NH}_{4}^{+}\right)$ entrant dans la composition minérale de l'eau inhibent davantage la sorption du ${ }^{137} \mathrm{Cs}$ que les ions bivalents tels $\mathrm{Ca}^{2+}$ et $\mathrm{Mg}^{2+}$. Lors d'expériences en batch avec des sédiments argileux limoneux à raison de $500 \mathrm{mg} \mathrm{l}^{-1}$ en eau distillée, une concentration de $100 \mathrm{mg} \mathrm{l}^{-1}$ en $\mathrm{K}^{+}$conduit à un $\mathrm{Kd}$ de 3 contre $10 \mathrm{~m}^{3} \mathrm{~kg} \mathrm{sec}^{-1}$ pour la même concentration en $\mathrm{Ca}^{2+}$ ou $\mathrm{Mg}^{2+}$ (Reynolds et Gloyna, 1963). Le césium ne forme pas significativement des complexes avec les ligands organiques dissous tels les acides humiques. Le $\mathrm{pH}$ dans sa gamme de variation observée dans les écosystèmes naturels (environ 4 à 8) n'affecte pas la sorption du césium. L'augmentation de la salinité de l'eau entraîne une diminution de la valeur du $\mathrm{Kd}$. La rétention du ${ }^{137} \mathrm{Cs}$ par la phase solide, diminue avec l'augmentation de la concentration en césium dissous seulement lorsque la quantité de césium ajoutée au milieu approche ou excède la quantité de césium stable échangeable du solide. Certains auteurs montrent que la répartition du césium entre les deux phases dépend de la proportion de radionucléide sous forme échangeable.

L'activité microbienne favorisée par les températures élevées $\left(25^{\circ} \mathrm{C}\right)$ serait à l'origine d'une sorption plus importante du césium par les sédiments riches en matières organiques. Les bactéries interviennent probablement sur le transfert entre l'eau et le sédiment de métaux traces, dont le césium.

Globalement, les valeurs conseillées dans les modèles globaux et revues bibliographiques relatives aux sédiments de surface varient de 0,05 à $80 \mathrm{~m}^{3} \mathrm{~kg}$ $\mathrm{sec}^{-1}$. Pour les matières en suspension, les valeurs s'étendent de 2 à $140 \mathrm{~m}^{3} \mathrm{~kg}$ 
J. GARNIER-LAPLACE $e t a l$.

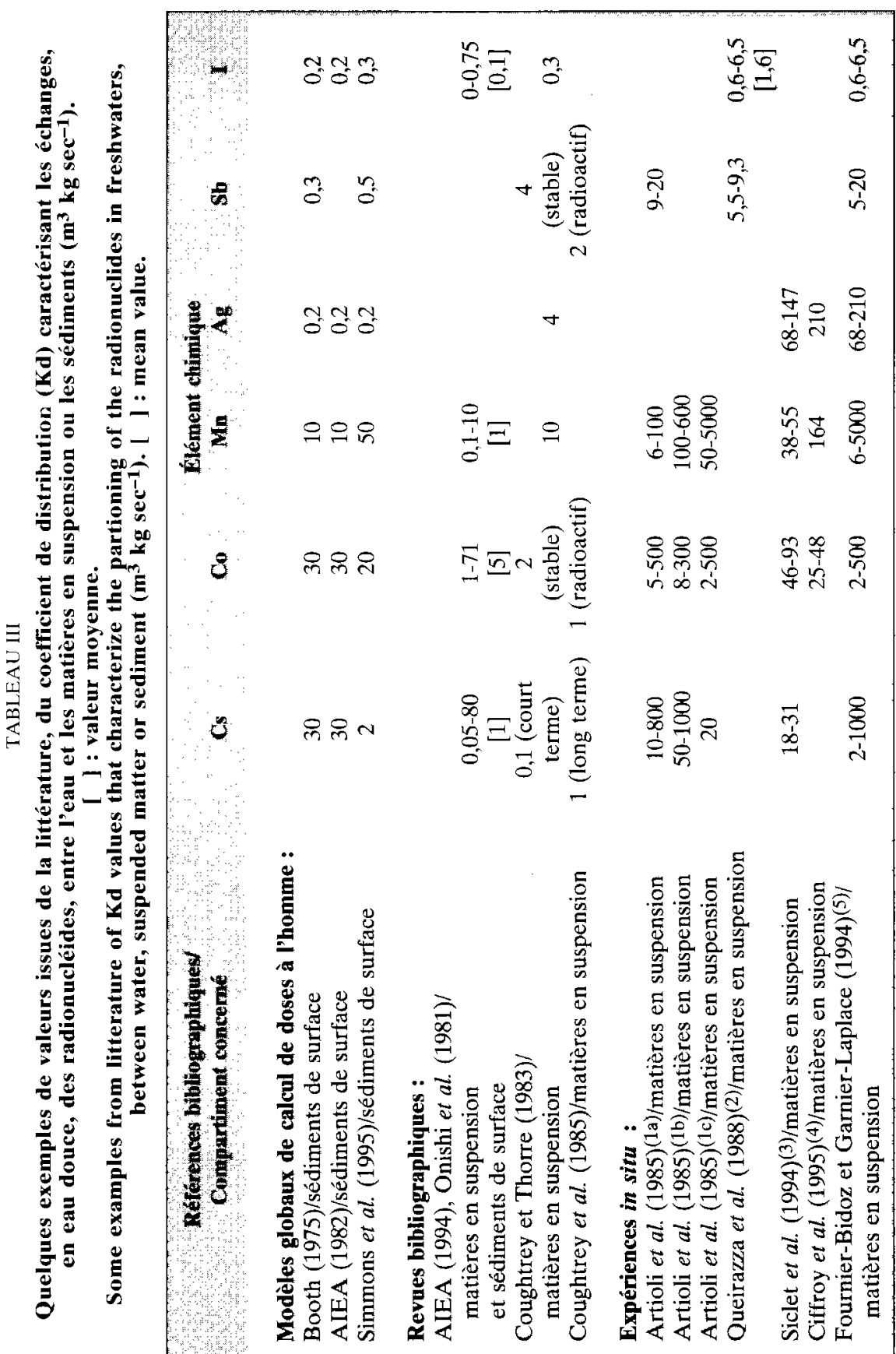


REJETS EN EAU DOUCE PAR LES CENTRALES NUCLÉAIRES

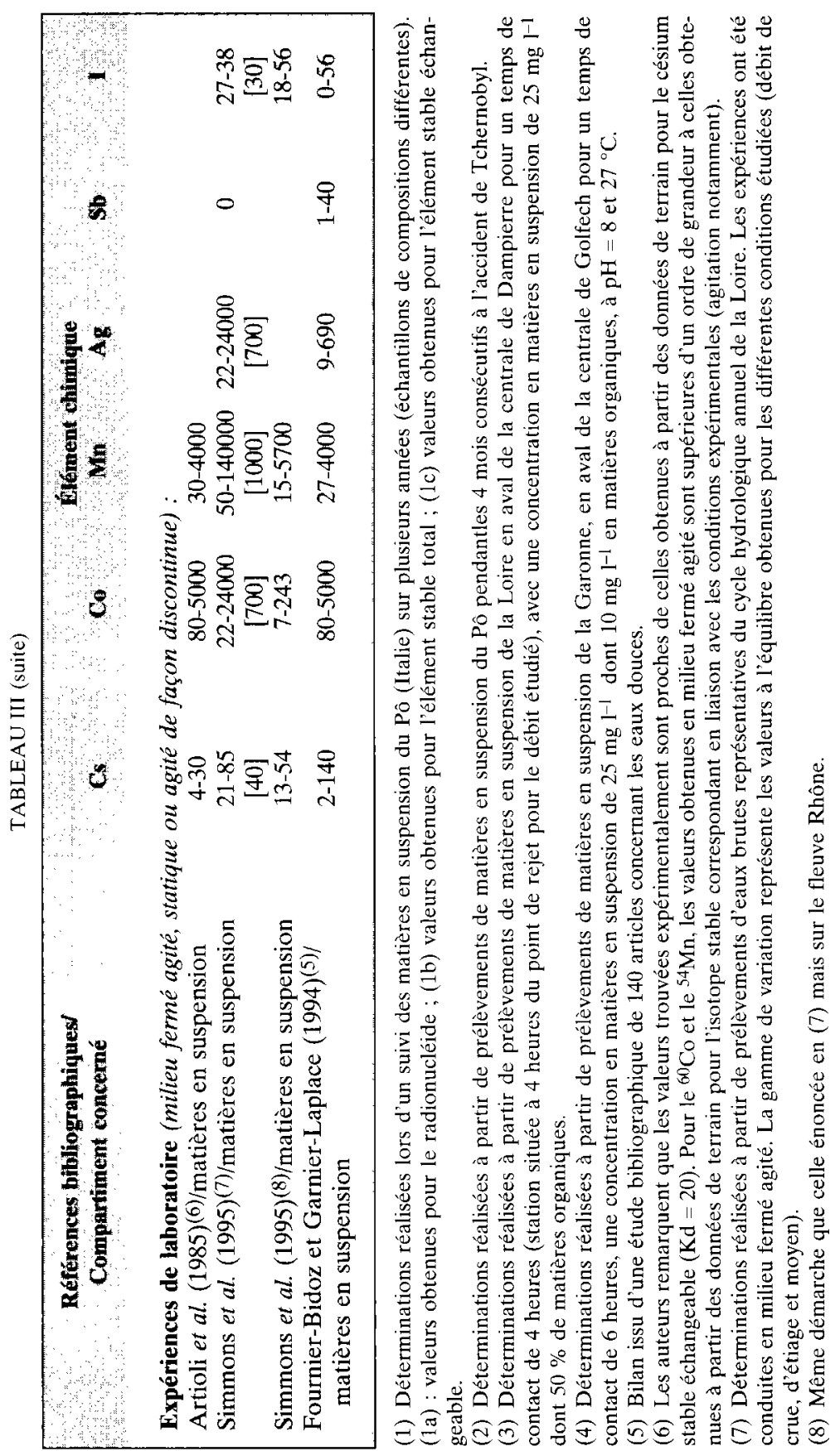


$\sec ^{-1}$ lorsqu'elles sont acquises en laboratoire. Sur le terrain, la gamme trouvée dans la littérature, présente une valeur maximale supérieure, s'étendant de 2 à $1000 \mathrm{~m}^{3} \mathrm{~kg} \mathrm{sec}^{-1}$.

\subsection{Cobalt}

\subsubsection{Mécanismes et cinétiques de sorption}

En liaison avec la présence dominante du cobalt sous forme d'ions cobaltineux $\left(\mathrm{Co}^{2+}\right)$ et de complexes solubles divers, plusieurs mécanismes de sorption, simultanés ou s'excluant, sont rapportés dans la littérature où une importance différente leur est accordée selon les auteurs:

- la coprécipitation avec les dioxydes de manganèse ou des carbonates,

- la sorption des complexes organiques du cobalt,

- l'adsorption sur des oxydes, des hydroxydes, des carbonates et les matières organiques,

- l'échange ionique.

L'intensité des liaisons du cobalt avec les matériaux solides est forte. La cinétique de sorption présente deux phases, une rapide de l'ordre de quelques heures à deux jours, pendant laquelle la sorption est importante, et une lente durant laquelle la quantité de cobalt fixée augmente lentement. Ces deux phases correspondent soit à deux réactions consécutives, soit à deux réactions parallèles. Le temps d'obtention de l'équilibre entre les deux phases déterminé lors d'expériences en milieu fermé (batch) agité, varie de 2 à 20 jours selon les auteurs.

\subsubsection{Influence des paramètres environnementaux}

Le temps de contact est un facteur important et l'équilibre entre phases solide et liquide n'est pas toujours atteint en raison de l'existence d'une étape lente dans la cinétique de sorption. L'évolution du $\mathrm{Kd}$ en fonction de la concentration en matières en suspension est variable selon le milieu étudié. L'évolution la plus fréquente serait la diminution du $\mathrm{Kd}$ avec l'augmentation de la concentration des matières en suspension mais l'influence de la concentration en matières en suspension sur le Kd dépend aussi du temps de contact. Lorsque ce dernier est suffisamment long, la diminution du Kd est observée uniquement pour les fortes concentrations. A l'inverse, pour les temps de contact courts, la valeur du $\mathrm{Kd}$ augmente avec la concentration quand celle-ci reste faible. A titre d'exemple, lors d'expériences en batch à partir de la fraction de diamètre inférieur à $0,1 \mathrm{~mm}$ de sédiments de surface simulant les matières en suspension (échantillons de la Dudvah, Slovaquie), les valeurs de $\mathrm{Kd}$ ont été estimées pour une gamme de concentration en matières en suspension allant de 30 à $8000 \mathrm{mg} \mathrm{l}^{-1}$ et pour $16 \mathrm{~h}$ et $32 \mathrm{j}$ de contact (Benes et al., 1989). Pour le temps de contact court, il se produit une augmentation du Kd de 5 à 110 $\mathrm{m}^{3} \mathrm{~kg} \mathrm{sec}^{-1}$ pour les concentrations en matières en suspension variant de 30 à 
$600 \mathrm{mg} \mathrm{l}^{-1}$ alors que la valeur reste stable (autour de $70 \mathrm{~m}^{3} \mathrm{~kg} \mathrm{sec}^{-1}$ ) pour les concentrations supérieures à $600 \mathrm{mg} \mathrm{1}^{-1}$. Pour le temps de contact long, la valeur du $\mathrm{Kd}$ reste constante, variant de manière non significative autour de $250 \mathrm{~m}^{3} \mathrm{~kg} \mathrm{sec}^{-1}$ de 8000 à $600 \mathrm{mg} \mathrm{l}^{-1}$ en matières en suspension, puis diminue continuellement jusqu'à la valeur de $35 \mathrm{~m}^{3} \mathrm{~kg} \mathrm{sec}^{-1}$ pour les concentrations en matières en suspension décroissante jusqu'à $30 \mathrm{mg} \mathrm{l}^{-1}$. L'effet de la composition chimique des matières en suspension est controversé. Selon certains auteurs, elle aurait une influence mineure sur le processus de sorption alors que, pour d'autres, une accumulation plus importante serait provoquée par des composants de la phase solide tels les minéraux argileux (dont l'illite et la montmorillonite), les matières organiques et les oxydes et hydroxydes de fer et de manganèse. L'importance relative de la rétention par ces composés dépend de certaines caractéristiques du milieu étudié qui sont le $\mathrm{pH}$, la composition de la phase aqueuse et la concentration en radionucléide. L'adsorption est plus importante sur les grains fins de taille inférieure à $0,1 \mathrm{~mm}$ que sur la fraction plus grossière $(<2 \mathrm{~mm})$. Le $\mathrm{pH}$ de la phase liquide est l'un des facteurs les plus importants de la rétention du cobalt en raison de son caractère hydrolysable. Le coefficient de distribution augmente avec le $\mathrm{pH}$ pour le domaine s'étendant de 4 à 8 environ, l'augmentation la plus importante se situant entre les valeurs 6 et 7,5 du pH. Au-delà de $\mathrm{pH}=8$, le $\mathrm{Kd}$ diminue avec l'augmentation du pH. Pour des échantillons du Var (Monaco) après $24 \mathrm{~h}$ de contact, pour une concentration en matières en suspension de $250 \mathrm{mg} \mathrm{l}^{-1}$ et une concentration en cobalt stable de $25 \mu \mathrm{g} \mathrm{\textrm {I } ^ { - 1 }}$, la valeur du $\mathrm{Kd}$ passe de 1 à $16 \mathrm{~m}^{3} \mathrm{~kg} \mathrm{sec}^{-1}$ entre $\mathrm{pH}=4$ à $\mathrm{pH}=8$ (Murray et Murray, 1973). La diminution de la sorption pour les $\mathrm{pH}$ supérieurs à 7,5-8, est attribuée à la formation de $\mathrm{Co}(\mathrm{OH})^{+}$ou de colloïdes s'associant peu au solide, à la compétition des ions $\mathrm{H}^{+}$avec les cations du cobalt pour la sorption sur les solides ou encore à des changements des propriétés de surface des particules. La composition chimique minérale des eaux douces semble avoir peu d'influence sur la fixation du cobalt. Les ions $\mathrm{Ca}^{2+}, \mathrm{Mg}^{2+}, \mathrm{Na}^{+}, \mathrm{K}^{+}$ou $\mathrm{NH}_{4}{ }^{+}$ne présentent pas d'effet significatif et la force ionique $(0,001$ à $0,01 \mathrm{M})$ a peu d'effet. Une diminution de la sorption n'est observée que pour les forces ioniques élevées $(0,1 \mathrm{M})$. Les composés organiques dissous ont une influence variable sur le $\mathrm{Kd}$ en fonction de leur nature. La présence de ligands polycycliques $\left(10^{-4}\right.$ à $\left.10^{-5} \mathrm{M}\right)$ de groupement fonctionnel varié (1-10-phénanthroline, 1-nitroso-2-naphtol) augmente sa valeur (respectivement pour chacun des composés cités, 168 et $358 \mathrm{~m}^{3} \mathrm{~kg}$ $\sec ^{-1}$ contre 3 en l'absence de ces composés) par adsorption sur les sédiments et complexation du cobalt (Sibley et al., 1981). Par contre, l'EDTA (concentration $>10^{-6} \mathrm{M}$ ) est à l'origine d'une diminution du Kd d'un facteur 10 environ du fait de la formation de complexes hydrosolubles. L'acide humique a un effet faible (légère augmentation) ou nul. Les concentrations en composés organiques des eaux douces naturelles sont généralement insuffisantes pour assurer la formation de complexes solubles entre le cobalt et les composés organiques. L'évolution typique du $\mathrm{Kd}$ avec la salinité est une diminution de la valeur du $\mathrm{Kd}$ lorsque la salinité augmente en liaison avec la formation de complexes. 
L'effet de la concentration en cobalt dissous est à prendre en compte uniquement si la quantité de cobalt ajoutée est comparable ou supérieure à la quantité de cobalt échangeable du solide. Il dépend donc de la nature et de la concentration en matières en suspension ce qui permet d'expliquer l'absence d'influence de la concentration en cobalt dissous constatée par d'autres auteurs.

Les résultats de travaux en laboratoire montrent que la fixation du ${ }^{60} \mathrm{Co}$ est plus importante en présence qu'en absence de microorganismes en raison de la bioaccumulation du cobalt. Lorsque cette activité biologique est inhibée, une diminution d'un facteur deux à dix de la valeur du Kd est obtenue. Les bactéries interviennent sur le transfert du cobalt entre l'eau et le sédiment avec une désorption du cobalt lorsque l'activité bactérienne est présente.

Globalement, les valeurs conseillées dans les modèles globaux et revues bibliographiques relatives aux sédiments de surface, varient de 1 à $71 \mathrm{~m}^{3} \mathrm{~kg}$ $\mathrm{sec}^{-1}$. Pour les matières en suspension, les valeurs s'étendent de 80 à $5000 \mathrm{~m}^{3} \mathrm{~kg} \mathrm{sec}^{-1}$ lorsqu'elles sont acquises en laboratoire. Sur le terrain, la gamme trouvée dans la littérature, va de 2 à $500 \mathrm{~m}^{3} \mathrm{~kg} \mathrm{sec}^{-1}$. Les valeurs importantes déterminées en laboratoire peuvent être dues à une coprécipitation ou adsorption du radionucléide sur des hydroxydes ou des carbonates d'autres éléments en quantité suffisante pour précipiter (manganèse, calcium...) dont la formation peut être favorisée par les conditions expérimentales telle l'agitation.

\subsection{Manganèse}

\subsubsection{Mécanismes et cinétiques de sorption}

Le mécanisme impliqué dans la sorption du manganèse est l'échange d'ions en raison de la forme dissoute dominante $\left(\mathrm{Mn}^{2+}\right)$. Les résultats d'extractions chimiques du ${ }^{54} \mathrm{Mn}$ associé à des sédiments montrent que l'adsorption physique, la coprécipitation avec des oxydes de fer et des hydroxydes de manganèse et l'association avec des molécules organiques pourraient intervenir. Des expériences en milieu fermé agité montrent que l'équilibre entre les deux phases est obtenu rapidement (après 10 à 30 heures) et que la sorption est importante durant les premières heures.

\subsubsection{Influence des paramètres environnementaux}

L'influence de la taille des particules est tout à fait classique. Le manganèse est associé principalement aux matières minérales de la phase particulaire. Dans le cas de sédiments du Rhône par exemple, l'affinité du radionucléide pour les matériaux minéraux est observée pour les fractions fines $(<20 \mu \mathrm{m})$ mais pour les fractions plus grossières ( $>63 \mu \mathrm{m}$ ), une partie s'associe aux matières organiques. Les données bibliographiques consultées dans la littérature concernant les eaux douces ne permettent pas de conclure quant à l'influence de la concentration des matières en suspension. 
Le coefficient de distribution du manganèse est particulièrement sensible aux conditions oxydantes ou réductrices du milieu. L'augmentation du Eh peut conduire à une augmentation du $\mathrm{Kd} \mathrm{du}$ fait de la formation d'hydroxydes de manganèse insolubles. La remobilisation de métaux stables, dont le manganèse, par des agents complexants dissous organiques ou minéraux, à partir de sédiments est faible. Moins de $1 \%$ à $7 \%$ sont libérés en solution selon l'agent complexant utilisé dont l'EDTA, les phosphates et les nitrates. Les données concernant l'influence de la salinité de l'eau sont contradictoires. En fait, des travaux menés sur l'estuaire de la Seine (France), montrent que l'évolution de la concentration en manganèse stable dissous avec la salinité de l'eau, varie selon la plage de salinité concernée avec une influence notable du phénomène de remise en suspension se produisant en estuaire. Celui-ci provoque un lavage du sédiment entraînant l'augmentation de la concentration en manganèse de l'eau par contribution de l'eau interstitielle plus concentrée que l'eau surnageante.

L'activité bactérienne influence la valeur du Kd. En milieu estuarien, des auteurs ont montré qu'elle catalysait l'oxydation du manganèse conduisant à sa précipitation sous forme de dioxyde $\left(\mathrm{MnO}_{2}\right)$. Par contre, en milieu anaérobie, des bactéries peuvent réduire $\mathrm{MnO}_{2}$, impliquant une dissolution et une augmentation du manganèse en solution.

Globalement, les valeurs conseillées dans les modèles globaux et revues bibliographiques relatives aux sédiments de surface varient de 0,1 à $10 \mathrm{~m}^{3} \mathrm{~kg}$ $\mathrm{sec}^{-1}$. Pour les matières en suspension, les valeurs s'étendent de 27 à $4000 \mathrm{~m}^{3} \mathrm{~kg} \mathrm{sec}^{-1}$ lorsqu'elles sont acquises en laboratoire. Sur le terrain, la gamme trouvée dans la littérature, va de 6 à $5000 \mathrm{~m}^{3} \mathrm{~kg} \mathrm{sec}^{-1}$. Cet élément a été peu étudié jusqu'à présent. Un effort de recherche est nécessaire pour comprendre son comportement en eau douce, d'autant que différents paramètres environnementaux semblent l'influencer.

\subsection{Argent}

\subsubsection{Mécanismes et cinétiques de sorption}

Les mécanismes de sorption ne sont pas clairement établis. La coprécipitation pourrait intervenir dans l'adsorption de l'argent en eau douce légèrement basique. Les réactions chimiques mises en jeu ne sont pas connues. Les phénomènes de complexation avec les ligands minéraux et organiques en eau douce sont inexistants ou non significatifs. Des expériences en milieu fermé agité montrent que la cinétique de sorption est rapide avec un équilibre atteint en dix heures.

\subsubsection{Influence des paramètres environnementaux}

De manière classique, le coefficient de distribution diminue lorsque la concentration des matières en suspension augmente. Cependant, la quantité d'argent adsorbée sur la phase solide augmente de façon non proportionnelle avec une 
quantité fixée par unité de masse de solide plus forte pour les faibles concentrations de matières en suspension. A titre d'exemple, le pourcentage d'argent fixé sur la phase solide dans les expériences conduites sur des échantillons du Var (Monaco) en batch à $\mathrm{pH}=8,1$ passe de $20 \%$ pour une concentration en matières en suspension de $100 \mathrm{mg} \mathrm{l}^{-1}$ à $70 \%$ pour $500 \mathrm{mg} \mathrm{l}^{-1}$ (Murray et Murray, 1973).

La valeur du $\mathrm{Kd}$ dépend de la nature des solides en suspension. Les plus fortes affinités de l'argent sont observées pour le dioxyde de manganèse (81 à $90 \%$ d'argent adsorbé), la montmorillonite (60\%), l'hydroxyde ferrique fraîchement précipité (59\%) et l'illite (14 à $28 \%$ ). La kaolinite et l'oxyde ferrique adsorbent respectivement 13 et $5 \%$ de l'argent en solution.

Le $\mathrm{pH}$ a une influence sur la rétention de l'argent par les matières en suspension. Une adsorption constante est observée jusqu'à $\mathrm{pH}=7$ (70 \% du radionucléide adsorbés et $\mathrm{Kd}=9,2 \mathrm{~m}^{3} \mathrm{~kg} \mathrm{sec}^{-1}$ ) et, au-delà, l'adsorption augmente $\left(85 \%\right.$ du radionucléide adsorbés à $\mathrm{pH}=8,5$ et $\left.\mathrm{Kd}=20,7 \mathrm{~m}^{3} \mathrm{~kg} \mathrm{sec}^{-1}\right)$. Comme pour le cobalt, ceci est à mettre en liaison avec la distribution des formes hydroxydes complexées de l'argent en fonction du $\mathrm{pH}$. Les concentrations des éléments majeurs ( $\mathrm{Na}, \mathrm{Cl}, \mathrm{Ca}$ et $\mathrm{Si}$ ) et celles en argent stable dans le milieu liquide n'influencent pas la valeur du Kd. En fonction de la concentration en argent de la solution, la quantité d'argent adsorbée sur les matières en suspension augmente jusqu'à un maximum correspondant à la saturation du solide. La valeur du Kd dépend donc de la nature et de la masse de solide.

L'effet de l'activité bactérienne est faible. L'ajout d'un antibiotique provoque une augmentation de seulement $3 \%$ de la fraction d'argent sous forme dissoute. L'influence de la présence de phytoplancton a également été largement démontrée, augmentant la valeur du Kd jusqu'à un facteur 1000 en fonction de la teneur et de la qualité du phytoplancton dans les matières en suspension totales.

Il existe peu de valeurs dans la littérature relatives à l'argent. Pour les sédiments de surface, Booth (1975) repris par l'AIEA (1982) et Simmons et al. (1995), estime sa valeur à $0,2 \mathrm{~m}^{3} \mathrm{~kg} \mathrm{sec}^{-1}$. Coughtrey et al. (1985) proposent à partir de leur revue bibliographique, une valeur plus élevée égale à $4 \mathrm{~m}^{3} \mathrm{~kg}$ $\mathrm{sec}^{-1}$. Pour les matières en suspension, les valeurs s'étendent de 9 à $690 \mathrm{~m}^{3} \mathrm{~kg}$ $\mathrm{sec}^{-1}$ lorsqu'elles sont acquises en laboratoire (bilan sur trois études) et de 68 à $210 \mathrm{~m}^{3} \mathrm{~kg} \mathrm{sec}^{-1}$ lorsqu'elles sont acquises sur le terrain. Simmons et al. (1995) suggèrent une gamme de variations identique à celle proposée pour le cobalt soit de 22 à $24000 \mathrm{~m}^{3} \mathrm{~kg} \mathrm{sec}^{-1}$.

\subsection{Antimoine}

\subsubsection{Mécanismes et cinétique de sorption}

Les études de l'interaction de l'antimoine avec les sédiments ou les matières en suspension des eaux douces sont peu nombreuses, vraisemblablement en raison 
de sa faible sorption liée à la forme dissoute dominante qui est l'ion antimoniate $\mathrm{Sb}(\mathrm{OH})_{6}{ }^{-}$. Aucun mécanisme de rétention n'est proposé dans la littérature. La sorption de l'antimoine, quand elle est observée, semble rapide.

\subsubsection{Influence des paramètres environnementaux}

Les paramètres environnementaux ont peu d'effet sur la sorption, l'antimoine restant en majorité sous forme dissoute $\left(\mathrm{Sb}(\mathrm{OH})_{6}{ }^{-}\right)$. Toutefois, l'influence du $\mathrm{pH}$ a été observée. Certains montrent qu'à $\mathrm{pH}=7,5$, une faible augmentation de ce paramètre $(0,2$ unité) entraîne une diminution du $\mathrm{Kd}$ (passage de 11 à $8 \mathrm{~m}^{3} \mathrm{~kg} \mathrm{sec}^{-1}$ ) indiquant la nature anionique de l'antimoine en solution. Les formes neutres et complexées solubles (complexes de l'antimoine stables et hydrosolubles formés par interaction avec des ligands minéraux ou organiques) dominent dans la plage de $\mathrm{pH} 4$ à 8 et sont à l'origine de la grande mobilité du composé dans les eaux. L'augmentation de la salinité de l'eau entraîne une diminution de la valeur du coefficient de distribution. Le $\mathrm{Kd}$ diminue de $10 \mathrm{~m}^{3} \mathrm{~kg} \mathrm{sec}^{-1}$ en eau douce (salinité proche de $0^{\circ} \%$ ) à $8 \mathrm{~m}^{3} \mathrm{~kg} \mathrm{sec}^{-1}$ en eau de mer (salinité de $29 \%$ ) (Li et al., 1984).

Les bactéries interviennent probablement sur le transfert entre l'eau et le sédiment de l'antimoine naturellement présent dans un écosystème d'eau douce. Pour des conditions telles que l'activité bactérienne soit significative, la sorption de l'antimoine par les sédiments est plus faible qu'en absence de l'activité bactérienne.

Globalement, les valeurs conseillées dans les modèles globaux et revues bibliographiques relatives aux sédiments de surface, s'étendent de 0,3 à $4 \mathrm{~m}^{3} \mathrm{~kg}$ $\mathrm{sec}^{-1}$. Pour les matières en suspension, les valeurs varient de 1 à $40 \mathrm{~m}^{3} \mathrm{~kg} \mathrm{sec}^{-1}$ qu'elles soient acquises en laboratoire ou sur le terrain. Les plus fortes valeurs semblent surprenantes en raison de la faible affinité du composé mise en évidence lors des quelques études disponibles dans la littérature. Elles demandent validation avant toute utilisation.

\subsection{Iode}

\subsubsection{Mécanismes et cinétiques de sorption}

Les formes solubles de l'iode en eau douce généralement proposées par les auteurs sont les iodures $\left(\mathrm{I}^{-}\right)$, les iodates $\left(\mathrm{IO}_{3}^{-}\right)$et les iodes organiques. Le mécanisme principal de sorption est l'adsorption sur les matières organiques en général. Selon Behrens (1985), l'activité microbienne dans les cours d'eau serait à l'origine d'une transformation des iodures en composés organiques iodés suite à une oxydation enzymatique suivie d'une incorporation de l'iode dans les composés organiques. Des expériences d'extractions chimiques de 1'131I fixé sur les sédiments confirment la forte affinité de l'iode avec les matières organiques mais montrent aussi qu'une fraction du radionucléide s'associe aux oxydes ou hydroxydes de fer et de manganèse. La cinétique de 
sorption de $\mathrm{l}^{\prime 131} I$ est rapide mais faible avec une concentration importante restant dans la solution (sorption maximale à partir de trois jours de contact). Une seule étude de laboratoire suggère l'existence d'une phase lente avec un équilibre non atteint en un mois.

\subsubsection{Influence des paramètres environnementaux}

L'influence de la nature de la phase solide n'a pas été étudiée en eau douce. A titre d'informations, dans les sols de $\mathrm{pH}=4$ à 6 , la fixation de l'iode sous forme iodure n'est pas corrélée avec le contenu en argile et en carbone organique mais avec la quantité de limon, en raison des charges positives développées par des couches d'oxydes de fer et d'aluminium amorphes. La fraction de l'iode sous forme organique $\left(\mathrm{ICH}_{3}\right)$ est corrélée au contenu en argile et en carbone organique des sols en raison des surfaces spécifiques élevées de ces matériaux et aux forces de liaison de Van der Waals de la matière organique. D'autres études montrent que iodures et iodates s'associent aux oxydes de fer et d'aluminium et à la matière organique avec une importance relative de ces différents composants variable selon le $\mathrm{pH}$ du sol. L'influence classique de la taille des particules en suspension est contestée par certains auteurs. Ceux-ci, observant la distribution de $\mathrm{l}^{131}$ I issu des retombées atmosphériques de l'accident de Tchernobyl (131I principalement lié aux particules de diamètre inférieur à $0,47 \mu \mathrm{m}$ ) montrent une redistribution du radionucléide dans le cours d'eau, au profit de particules plus grossières (diamètre supérieur à $45 \mu \mathrm{m}$ ).

Le $\mathrm{pH}$, le potentiel redox et les diverses matières organiques de l'eau influent sur les formes chimiques de l'iode. Cependant, quelle que soit sa forme, la sorption apparaît faible. Son adsorption sous forme anionique (iodure et iodate) est particulièrement limitée pour le domaine 6 à $8 \mathrm{du} \mathrm{pH}$ en raison de la faible capacité d'échange anionique de la majorité des sédiments. Des expériences en milieu fermé agité montrent qu'une variation de $\mathrm{pH}$ entre 6 et 9 n'affecte pas la sorption de l'131I par la phase solide. Une fraction de l'iode associé aux sédiments en eau douce est susceptible d'être libérée dans la phase liquide au contact de l'eau saline.

Globalement, les valeurs de la littérature relatives aux sédiments varient de 0 à $1 \mathrm{~m}^{3} \mathrm{~kg} \mathrm{sec}^{-1}$. Pour les matières en suspension, les rares valeurs disponibles s'étendent de 0 à $56 \mathrm{~m}^{3} \mathrm{~kg} \mathrm{sec}-1$ avec des valeurs semblant plus élevées en laboratoire que sur le terrain.

\section{Démarche proposée pour l'estimation des cinétiques d'échange entre l'eau et les matières en suspension pour un écosystème précis}

Pour un écosystème donné, il n'existe pas une valeur de Kd par radionucléide mais une gamme de variation dont l'étendue est plus ou moins corrélée, selon le radionucléide, à l'étendue des valeurs prises au cours d'un cycle hydrolo- 
gique annuel par les paramètres physiques, chimiques et biologiques énumérés au tableau I. La démarche préconisée est d'analyser le plus précisément possible les variations saisonnières des paramètres majeurs afin de mettre en évidence une ou plusieurs tendances en relation avec la courbe de distribution des débits observée au cours d'un cycle hydrologique. Dans la plupart des cours d'eau de zone tempérée, trois tendances dominantes peuvent souvent. être dégagées ; une tendance représentative des débits d'étiage, influencée par la présence notable de phytoplancton (modification de la composition des matières en suspension, augmentation du $\mathrm{pH}$...), une tendance représentative des débits de crue, caractérisée essentiellement par une concentration élevée en matières en suspension et une tendance «moyenne » (débits moyens) pour le reste de l'année. Une analyse des données bibliographiques, orientées par la connaissance des gammes de variations de ces paramètres, permet ensuite de réduire les vastes gammes de variations données dans la littérature aux conditions représentatives de l'écosystème considéré. En cas de spécificité pour le cours d'eau étudié telle une forte concentration en matières organiques solubles par exemple, une approche expérimentale (terrain et/ou laboratoire) conduite selon un protocole défini précis, permettra d'évaluer les valeurs de $\mathrm{Kd}$ en fonction du temps (variations saisonnières) et de l'espace (influence du temps de contact) propres à l'écosystème.

Une approche en laboratoire peut être conduite selon une infinité de protocoles. Le choix de ce type de démarche ne doit s'effectuer qu'après définition d'un protocole rigoureux, choisi selon les objectifs à atteindre, le plus représentatif des conditions dans lesquelles on souhaite appliquer la valeur du Kd obtenue. L'étape de validation sur le terrain sera toujours vivement souhaitable. La technique du milieu fermé (batch) non agité est séduisante car rapide, simple, donnant des informations sur la distribution du radionucléide dans ce contexte expérimental précis. Elle devra être complétée par une étude de l'influence de l'agitation afin de cerner les éventuels effets liés aux conditions hydrodynamiques existant dans un système naturel. Il demeure impératif, durant ce type d'expériences, de contrôler les paramètres majeurs influençant les valeurs de $\mathrm{Kd}$, notamment le $\mathrm{pH}$ et le potentiel redox. La composition en anions et cations majeurs doit être suivie tout au long de l'expérience et les matières en suspension doivent être parfaitement caractérisées (granulométrie, minéralogie, matières organiques, phytoplancton). Il est également impératif de connaître la concentration en élément stable et radioactif, en essayant de travailler, dans la mesure du possible, avec des sources radioactives de forme chimique parfaitement identifiée. Enfin, il semble essentiel de collecter des échantillons d'eau brute représentatifs du cycle hydrologique du cours d'eau (optimisation du mode de prélèvement), de les transporter rapidement au laboratoire dans les meilleures conditions $\left(4^{\circ} \mathrm{C}\right.$, obscurité $)$, et de réduire au mieux le délai entre prélèvement et expériences afin d'éviter les évolutions des deux phases en présence.

Étant donnée la complexité de la démarche expérimentale en laboratoire par le nombre de paramètres importants à suivre et à fixer $(\mathrm{pH}$, potentiel 
redox, concentration en matières en suspension et composition de la phase solide, concentration en élément stable, minéralisation de l'eau, concentration en matières organiques dissoutes...), l'approche «terrain » semble beaucoup plus séduisante, à condition d'acquérir également les informations précédemment citées. Cette approche nécessite un échantillonnage d'eau brute au minimum saisonnier, de façon à cerner l'ensemble des variations se produisant dans le cours d'eau au cours d'un cycle annuel. Cet échantillonnage devra être effectué également en plusieurs stations au fil de l'eau de manière à mettre en évidence l'influence du temps de contact. Cependant, cette approche présente des limites dont les principales sont les suivantes :

- il faut que la centrale située sur le cours d'eau à étudier puisse être en mesure de rejeter des effluents ;

- les concentrations des radionucléides dans l'eau doivent être modélisées sur la base d'un certain nombre d'hypothèses dont la validité peut être discutée ;

- certains radionucléides ne sont pas détectables en raison de leur concentration trop faible ou de leur faible fixation au niveau des particules solides ;

- si l'écosystème a subi une contamination antérieure (retombées anciennes et accident de Tchernobyl pour le ${ }^{137} \mathrm{Cs}$, rejets d'effluents liquides de centrales pour les huit radionucléides identifiés), plusieurs formes du radioélément pas forcément échangeables, peuvent coexister.

$\mathrm{Au}$ bilan aucune des deux démarches, études en laboratoire et in situ, n'apparaît comme indubitablement la meilleure et, en fait, elles sont totalement complémentaires.

\section{Conclusion générale}

Dans le domaine des eaux douces, un très grand nombre de travaux existent concernant les échanges des radionucléides entre phases solides et liquide constituant les compartiments physiques des écosystèmes naturels. Les travaux concernant les isotopes radioactifs du césium et du cobalt sont nombreux alors que les études portant sur les autres radionucléides sont plus rares (manganèse, argent), voire quasiment inexistants (antimoine, iode). L'analyse bibliographique réalisée à partir de plus d'une centaine d'articles sur le sujet (FournierBidoz et Garnier-Laplace, 1994) démontre l'existence, pour un radionucléide donné, de nombreuses conclusions, souvent différentes, voire même contradictoires. En ce qui concerne les matières en suspension, l'examen du tableau III révèle des gammes de variations très étendues (différence jusqu'à un facteur $10^{3}$ entre les valeurs minimales et maximales) pour le cobalt, le manganèse et l'argent, étendues (différence jusqu'à un facteur $5 \times 10^{2}$ entre valeurs minimales et maximales) pour le $\mathrm{Cs}$, peu étendues pour l'antimoine et l'iode (différence jusqu'à un facteur 40 entre valeurs minimales et maximales). L'amplitude de ces gammes de variations est à mettre en relation avec le nombre de paramètres environnementaux influant sur la valeur du Kd et l'importance quantitative de cette influence. Pour le cobalt, le manganèse et l'argent, les valeurs 
maximales obtenues dans le cadre des expériences de laboratoire sont souvent supérieures d'un ordre de grandeur à celles obtenues à partir des données de terrain, en liaison avec des problèmes méthodologiques tels que par exemple, la formation d'hydroxydes et/ou de carbonates insolubles dans le milieu expérimental en général agité, voire aéré. En ce qui concerne les sédiments de surface, les valeurs conseillées dans les modèles globaux de calcul de doses à l'homme sont plus élevées d'un facteur 10 pour le cobalt, le manganèse et le césium que pour l'argent, l'antimoine et l'iode (Tab. III). L'analyse bibliographique a permis de dégager, pour chaque radionucléide, un ou plusieurs paramètres environnementaux dont l'influence peut être jugée majeure (Tab. IV). Pour chaque élément, il demeure essentiel de considérer le caractère spécifique, à chaque écosystème à un moment donné de son cycle hydrologique (et écologique), de la valeur du coefficient de distribution. Une valeur précise de $\mathrm{Kd}$ ne doit donc être utilisée au sein d'un modèle de calcul de dose qu'après s'être assuré de l'adéquation, pour les paramètres majeurs influençant sa détermination, des conditions d'obtention de ce coefficient de distribution et des conditions imposées par le scénario choisi pour initialiser le modèle.

\section{TABLEAUIV}

Paramètres environnementaux majeurs dont la connaissance au sein de l'écosystème étudié, est impérative pour un usage correct du coefficient de distribution (adéquation des conditions d'obtention et d'application de la valeur).

Major environmental parameters that must be known for the studied natural ecosystem in the aim to use correctly the value of Kd (correspondence between conditions under which the value has been obtained and conditions under which the value will be applied).

\begin{tabular}{|c|c|c|c|c|c|c|}
\hline Paramètres environnementaux & $134 \mathrm{Cs}$ & ${ }^{58} \mathrm{Ca}$ & $\begin{array}{l}\text { Radion } \\
\mathbf{5 4} \mathbf{M n}\end{array}$ & nocléide. & $12 \mathrm{sb}$ & 1311 \\
\hline Concentration en matières en suspension & $\square$ & $\square$ & $\square$ & $\square$ & $\square$ & $\square$ \\
\hline Temps de contact & $\square$ & $\square$ & $\square$ & $\square$ & $\square$ & $\neg$ \\
\hline Qualité de la phase solide & $\square$ & $\square$ & $\square$ & $\square$ & & $\square$ \\
\hline Granulométrie de la phase solide & $\square$ & $\square$ & $\square$ & $\square$ & & $\neg$ \\
\hline $\begin{array}{l}\text { Concentration en élément stable de l'eau } \\
\text { et de la phase solide }\end{array}$ & $\square$ & $\square$ & $\square$ & $\square$ & & \\
\hline $\begin{array}{l}\text { Conditions d'oxydoréduction de l'eau } \\
\text { et de la phase solide }\end{array}$ & $\square$ & & $\square$ & & & \\
\hline $\begin{array}{l}\text { Concentration en matières organiques } \\
\text { dissoutes et qualité }\end{array}$ & & $\square$ & & & $\square$ & $\square$ \\
\hline pH de l'eau & & $\square$ & $\square$ & $\square$ & & \\
\hline Composition minérale de l'eau & $\square$ & & & & & \\
\hline Activité des microorganismes & $\square$ & $\square$ & $\square$ & & $\square$ & \\
\hline
\end{tabular}




\section{RÉFÉRENCES}

AIEA (1982) Generic models and parameters for assessing the environmental transfer of radionuclides from routine releases. Exposures of critical groups, Safety series $\mathbf{n}^{\circ} 57$. AIEA, Vienne.

AIEA (1985) Hydrological dispersion of radioactive material in relation to nuclear power plant siting. A safety guide, Safety series $n^{\circ}$ 50-SG-S6. AIEA, Vienne.

AIEA (1994) Handbook of parameter values for the prediction of radionuclide transfer in temperate environments. Technical report series $n^{\circ} 364$. AIEA, Vienne.

Artioli R., Ciceri G., Queirazza G., Traversi A.L. (1985) Radionuclide partitioning between water and sediment (bottom and suspended): comparison of different methodologies. In: Third Research Coordination Meeting of the coordinated research programme on the role of sediments in transport and accumulation of radionuclides in waterways, December 2-7, 1985, La Casaccia, Rome. 23 p. Publications office, Milan, CISE 2919.

Behrens H. (1985) Speciation of radioiodine in aquatic and terrestrial systems under influence of biogeochemical processes. In: Speciation of fission and activation products in the environment, Oxford, April 16-19, 1985 (Bulman, Cooper, Eds). Elsevier Appl. Sci., Londres, New-York.

Benes P., Jurak M., Cernik M. (1989) Factors affecting interaction of radiocobalt with river sediment. II. Composition and concentration of sediment, temperature. J. Radioanal. Nucl. Chem., 133, 225-239.

Booth R.S. (1975) A system analysis model for calculating radionuclide transport between receiving waters and bottom sediments (Environmental Sciences Division Publication $\mathrm{n}^{\circ}$ 705). ORNL, Oak Ridge.

Ciffroy P., Siclet F., Humbert B. (1995) In situ determination of $110 \mathrm{~m} A g,{ }^{58} \mathrm{Co},{ }^{60} \mathrm{Co}$ and ${ }^{54} \mathrm{Mn}$ distribution between freshwater and suspended matter. In: Internat. Symp. on environnemental impact of radioactive releases, 8-12 mai, 1995, Vienne. IAEA-SM339, Vienne.

Coughtrey P.J., Thorne M.C. (1983) Radionuclide distribution and transport in terrestrial and aquatic ecosystems. A critical review of data. Balkema A.A. publishers, Rotterdam.

Coughtrey P.J., Jackson D., Thorne M.C. (1985) Radionuclide distribution and transport in terrestrial and aquatic ecosystems. A compendium of data. Balkema A.A. publishers, Rotterdam.

Dzombak D.A., Ali M.A. (1993) Hydrochemical modeling of metal fate and transport in freshwater environment. Water Poll. Res. J. Canada, 28, 7-50.

Fournier-Bidoz V., Garnier-Laplace J. (1994) Étude bibliographique sur les échanges entre l'eau, les matières en suspension et les sédiments des principaux radionucléides rejetés par les centrales nucléaires (rapport SERE 94/073 (P)). IPSN/DPEI, Cadarache.

Håkanson L., Peters R.H. (1995) Predictive limnology : methods for predictive modelling. SPB Acad. publishing, Amsterdam.

Kharkar D.P., Turekian K.K., Bertine K.K. (1968) Stream supply of dissolved silver, molybdenum, antimony, selenium, chromium, cobalt, rubidium and cesium to the oceans. Geochim. Cos. Acta, 32, 285298.

Li Y.H., Burkhardt L., Teraoka H. (1984) Desorption and coagulation of trace elements during estuarine mixing. Geochim. Cos. Acta, 48, 1879-1884.

Murray C.N., Murray L. (1973) Adsorption-desorption equilibria of some radionuclides in sediment freshwater and sediment seawater systems. In: Radioactive contamination of the marine environment, july 10-14, 1972, Seattle. IAEA, Vienne.

Onishi Y., Serne R. J., Arnold E. M., Cowan C. E., Thompson F. L. (1981) Critical review: radionuclide transport, sediment transport, and water quality mathematical mode- 
ling and radionuclide adsorption/desorption mechanisms (NUREG/CR 1322, PNL 2901). Pacific Northwest Laboratory, Richland.

Picat P., Debeuns G., Maubert H., Cartier Y., Lacroix D., Angeli A., Diraison J., Caudoux B., Tempier C. (1979) La radioécologie appliquée aux études des sites électronucléaires. Etude radioécologique du Rhône moyen. Première partie : essai d'interprétation des mesures in situ de l'activité des sédiments. In: IIème Symposium Internatial de Radioécologie, 19-22 juin, 1979, Cadarache. IPSN, Cadarache.

Queirazza G., Guzzi L., Cigna A.A. (1988) Radionuclides behaviour in the Pô river ecosystem (N. Italy) after the Chernobyl accident. In: Impacts des accidents d'origine nucléaire sur l'environnement, IVème Symposium International de Radioécologie, 14-18 mars, 1988, Cadarache. IPSN, Cadarache.

Reynolds T.D., Gloyna E.F. (1963) Radioactivity transport in water Transport of strontium and cesium by strem and estuarine sediments. Technical report- 1 to the USAEC.

Sibley T.H., Sanchez A.L., Schell W.R. (1981) Distribution coefficients for radionuclides in aquatic environments. Adsorption studies of cobalt. College of fisheries, University of Washington, NUREG/CR-1852, 6.

Siclet F., Moisa D.A., Hita A., Ciffroy P., Gosse P. (1994) The role of suspended solids in the migration of radionuclides in rivers. In: Int. Seminar on freshwater and estuarine radioecology, mars $21-25$, Lisbonne.

Simmons J.R., Lawson G., Mayall A. (1995) Methodology for assessing the radiological consequences of routine releases to the environment, Rad. Prot., 72, Report EUR 15760 EN, European commission, Luxembourg. 